\title{
Influência do nível de atividade física e do gesto motor empregado durante procedimentos odontológicos na prevalência de dores localizadas em estudantes e professores de Odontologia
}

\section{Influence of the level of physical activity and the motor gesture used during dental procedures in the prevalence of localized pain in students and teachers of Dentistry}

Lucas da Silva Spinelli', Fabiano de Sant'Ana dos Santos ${ }^{3}$, Mateus Machado Delfino ${ }^{4}$, Vera Maria Cury Salemi², Douglas Pinheiro Miranda ${ }^{1,2}$

1- Laboratório de Avaliação Física e Fisiologia do Exercício (LAFFEX), Curso de Educação Física, Centro Universitário da Fundação Educacional de Barretos, Barretos, SP, Brasil.

2- Programa de Doutorado em Cardiologia, Instituto do Coração, Faculdade de Medicina da Universidade de São Paulo, São Paulo, SP, Brasil.

3- Professor Doutor de Orientação Profissional do Curso de Odontologia, Centro Universitário da Fundação Educacional de Barretos, Barretos, SP, Brasil.

4 - Doutorando em Odontologia pela Faculdade de Odontologia de Araraquara, Universidade Estadual Paulista, Araraquara, SP, Brasil.

\begin{abstract}
Resumo
O profissional cirurgião-dentista está entre os trabalhadores que mais sofrem com distúrbios musculoesqueléticos. Isso se deve a vários fatores, como postura inadequada, estilo de vida e gestos repetitivos. O objetivo do presente estudo foi investigar os agentes precursores de dores localizadas em alunos e professores de uma clínica odontológica. Participaram do estudo 148 voluntários, homens e mulheres, alunos e professores, com idade entre 17 a 63 anos (22,44 7,56 anos). Foi aplicado um questionário com o desenho do corpo humano, em que assinalavam o local que sentiam dores frequentes e a versão curta do International Physical Activity Questionnaire (IPAQ) para determinar o nível de atividade física. Também foi feita uma análise da postura de trabalho durante cinco atendimentos por meio de filmagens e fotografias, classificadas pelo método Rapid Upper Limb Assessment (RULA). Os resultados mostraram que a região em que sentiam mais dores era a região paravertebral. Apenas $4,73 \%$ dos voluntários tiveram um nível de atividade física adequado, enquanto $38,51 \%$ foram classificados com um nível irregular, porém os indivíduos mais ativos fisicamente apresentaram um nível de dores menor em relação aos menos ativos fisicamente. Em relação à postura de trabalho, a maior predominância indica que é necessário investigar e que podem ser necessárias mudanças. Conclusão: a região paravertebral mostrou ser mais propícia às dores localizadas nos estudantes e professores. Indivíduos mais ativos fisicamente apresentaram um menor nível de dores em relação aos menos ativos.
\end{abstract}

Palavras-chave: odontologia, ergonomia, exercício físico, saúde.

\footnotetext{
Autor correspondente: Douglas Pinheiro Miranda Av. Prof Roberto Frade Monte, 386 Barretos, SP, Brasil Cep:14783-226 e-mail: douglas@pinheiromiranda.com
}

Recebido em: 18/10/2018

Aceito para publicação em: 10/09/2019

https://doi.org/10.4322/1980-0029.032020 


\begin{abstract}
The professional dental surgeon is among the workers who suffer most from musculoskeletal disorders. This is due to several factors, such as inadequate posture, lifestyle and repetitive gestures. The objective of the present study was to investigate the agents' precursors of pain located in students and teachers of a dental clinic. A total of 148 volunteers, men and women, students and teachers, aged between 17 and 63 years participated in the study $(22,44 \pm 7,56$ years). A questionnaire was applied with the design of the human body, where they indicated the place that they had frequent pains and the short version of the International Physical Activity Questionnaire (IPAQ) to determine the level of physical activity. An analysis of the work posture was carried out during five visits through filming and photographs, classified by Rapid Upper Limb Assessment (RULA). The results showed that the region where they had the most pain was the vertebral region. Only $4.73 \%$ of the volunteers had an adequate level of physical activity, while $38.51 \%$ were classified with an irregular level, however the more physically active individuals presented a lower level of pain than the less physically active ones. In relation to the work posture, the greater predominance indicates that it is necessary to investigate and that changes may be necessary. The paravertebral region was shown to be more propitious to localized pain in students and teachers. More physically active individuals had a lower level of pain than the less active ones.
\end{abstract}

Keywords: Dentistry, Ergonomics, Physical Exercise, Health.

\section{Introdução}

Distúrbios musculoesqueléticos são um dos problemas de saúde mais comuns entre a população trabalhadora, os quais são frequentemente vistos em cirurgiões-dentistas (Saxena et al., 2013; Dantas \& Lima, 2015). Na Odontologia o trabalho é de cunho sedentário, estático e com muita utilização dos membros superiores, o que pode resultar em sobrecargas (Garcia \& Campos, 2013). Sua tarefa exige a realização de gestos motores repetitivos, esforços excessivos e posturas inadequadas, podendo ocorrer Lesões por Esforços Repetitivos (LER) ou Distúrbios Osteomusculares Relacionados ao Trabalho (DORT), o que pode comprometer a qualidade de vida desses profissionais (Souza et al., 2012).

Considerando que a postura corporal é um dos principais fatores de risco para distúrbios musculoesqueléticos na Odontologia, é importante averiguar as posturas de trabalho. Na literatura existem alguns métodos de avaliação postural, dentre eles o Rapid Upper Limb Assessment (RULA), recomendado pela International Standard Organization - ISO 11228. O RULA oferece uma análise rápida da postura sem interferir no trabalho do indivíduo (Garcia \& Campos, 2013). Outro fator contribuinte é a inatividade física. $\mathrm{Na}$ Índia 40,8\% dos cirurgiões-dentistas levam um estilo de vida sedentário, tendo maior risco de desenvolver doenças crônicas (Thakar et al., 2015). Portanto, se faz importante investigar o nível de atividade física dos voluntários. O objetivo do estudo foi investigar os agentes precursores de dores localizadas em alunos e professores da clínica odontológica do Centro Universitário da Fundação Educacional de Barretos (Unifeb), tais como nível de dores localizadas, nível de atividade física e avaliação biomecânica.

\section{Material e Métodos}

\section{Amostra}

O protocolo da presente pesquisa foi aprovado pelo Comitê de Ética e Pesquisa do Unifeb (CAAE: 17774513.9.0000.5433). Foram convidados a participar todos os alunos e professores do curso de Odontologia do Unifeb. A participação foi voluntária e confirmada após a assinatura do Termo de Consentimento Livre e Esclarecido (TCLE). Do total de convidados, 148 voluntários se propuseram a participar da pesquisa, sendo oito professores $(5,41 \%), 38$ alunos homens $(25,68 \%)$ e 102 alunas mulheres $(68,92 \%)$, com idade entre 17 a 63 anos (22,44 7,56 anos). Dentre os alunos, 53 são do primeiro ano de graduação, 44 são do segundo ano, dez são do terceiro ano e 33 são do quarto ano.

\section{Avaliação da prevalência de dores localizadas}

Os participantes receberam uma folha com a ilustração da parte anterior e posterior do corpo humano de acordo com a posição anatômica, 
em que eles assinalavam com um " $x$ " as regiões do corpo em que sentiam dores com frequência (a ilustração pode ser observada na Figura 1). Os dados foram analisados estatisticamente pelo software Microsoft Office Excel 2007.

\section{Avaliação do Nível de Atividade Física}

Para avaliar o nível de atividade física foi aplicado o International Physical Activity Questionnaire (IPAQ) em sua versão curta, validado no Brasil pelo Centro de Estudos do Laboratório de Aptidão Física de São Caetano do Sul - CELAFISCS (Matsudo et al., 2001). Os dados foram analisados pelo software Microsoft Office Excel 2007.

\section{Avaliação biomecânica}

A avaliação biomecânica foi realizada por meio de filmagens e registros fotográficos dos gestos motores empregados pelos dentistas durante o atendimento, com a preservação da imagem e sigilo da identidade tanto do dentista quanto do paciente. Apenas alunos que desenvolvem atividades na clínica odontológica poderiam participar desta avaliação. A atividade de atendimento começa a partir do segundo ano de graduação.
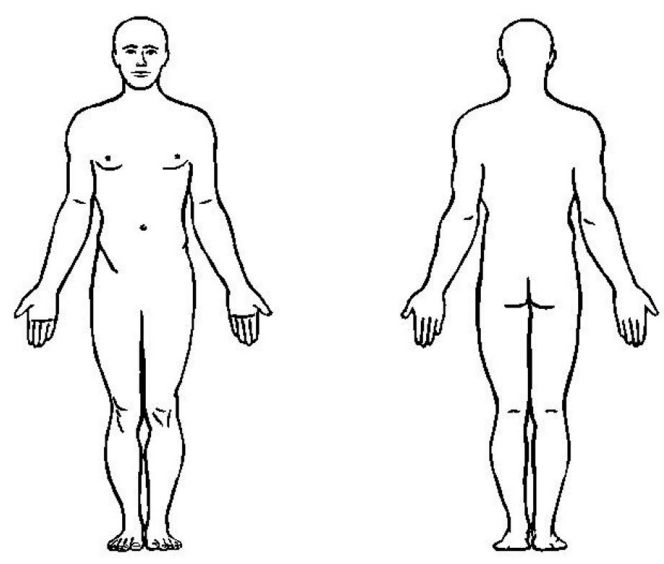

Figura 1. Questionário de dores localizadas.
A câmera utilizada foi o modelo semiprofissional Nikon Coolpix L820 e foi posicionada de acordo com cada procedimento odontológico por acontecerem em locais diferentes. Os procedimentos odontológicos analisados foram: raspagem radicular, instrumentação e obturação do sistema de canais radiculares, cimentação de coroas protéticas e exodontias. Todos os voluntários foram filmados e fotografados durante o período de atendimento. A cada atendimento realizado, realizou-se a troca do voluntário. Durante o procedimento de raspagem radicular a câmera foi posicionada a $45 \mathrm{~cm}$ de altura e 1,65 m de distância do centro da cadeira do paciente. A duração do atendimento foi aproximadamente 20 minutos. No momento da instrumentação do sistema de canais radiculares, a posição da câmera foi de $45 \mathrm{~cm}$ de altura e 2,10 m de distância, com tempo aproximado de 80 minutos. Na obturação do sistema de canais radiculares a câmera foi posicionada a $66 \mathrm{~cm}$ de altura e 1,92 m de distância. A duração foi de aproximadamente 75 minutos. Para a cimentação de coroas protéticas, a câmera ficou posicionada a $45 \mathrm{~cm}$ de altura e 2,05 de distância, com tempo aproximado de 35 minutos. No procedimento de exodontia a câmera foi posicionada a $92 \mathrm{~cm}$ de altura e 1,70 m de distância, com tempo aproximado de 65 minutos.

Para mensuração dos ângulos das articulações do corpo foi utilizado o software Kinovea 0.8.15 O método utilizado para classificar os ângulos articulares foi o Rapid Upper Limb Assessment (RULA), instrumento proposto por McAtamney \& Corlett (1993). Esse método de avaliação rápida foi desenvolvido para investigar a exposição dos trabalhadores aos fatores de risco associados aos distúrbios dos membros superiores ${ }^{7}$. Ferramenta que apresenta diversos scores, quanto mais alto o nível do score, maior a probabilidade para o desenvolvimento de lesões músculo esqueléticas, cada score supõe um tipo de intervenção a ser feita, observados na Tabela 1.

Tabela 1. Score final do protocolo RULA (Iwuala et al., 2015).

\begin{tabular}{ccc}
\hline Pontuação & Nível de ação & Intervenção \\
\hline 1 ou 2 & 1 & Postura aceitável, desde que não seja mantida por longos períodos. \\
3 ou 4 & 2 & É necessário investigar. Podem ser necessárias mudanças. \\
5 ou 6 & 3 & É necessário mudar logo. \\
7 & 4 & É necessário investigar e mudar imediatamente. \\
\hline
\end{tabular}




\section{Resultados}

\section{Prevalência de dores localizadas}

Os dados mostraram que as regiões mais apontadas com dores foram a região da coluna torácica, totalizando $26,35 \%$ dos voluntários, seguido da região da coluna lombar com $22,97 \%$, região da coluna cervical com $18,92 \%$, região anterior do joelho com $12,84 \%$ e a região sacral com $8,78 \%$. No Gráfico 1 são apresentadas todas as regiões analisadas e seus percentuais.

Dos participantes que relataram dores na região da coluna torácica, $28,21 \%$ são homens e $71,79 \%$ mulheres. Região da coluna lombar, $23,53 \%$ são homens e $76,47 \%$, mulheres. Na região da coluna cervical, $100 \%$ são mulheres.

Os professores relataram uma região com maior prevalência, a região sacral (25\%). Outras regiões tiveram percentuais iguais: coluna torácica $(12,50 \%)$, coluna lombar $(12,50 \%)$, coxa $(12,50 \%)$ e pés e dedos $(12,50 \%)$. Nas demais regiões não houve queixa de dor. Em média, apresentaram 0,75 pontos de dores. Entre os alunos do primeiro ano de graduação, as regiões mais apontadas foram coluna torácica $(24,53 \%)$, coluna cervical $(20,75 \%)$ e coluna lombar $(18,87 \%)$, representando em

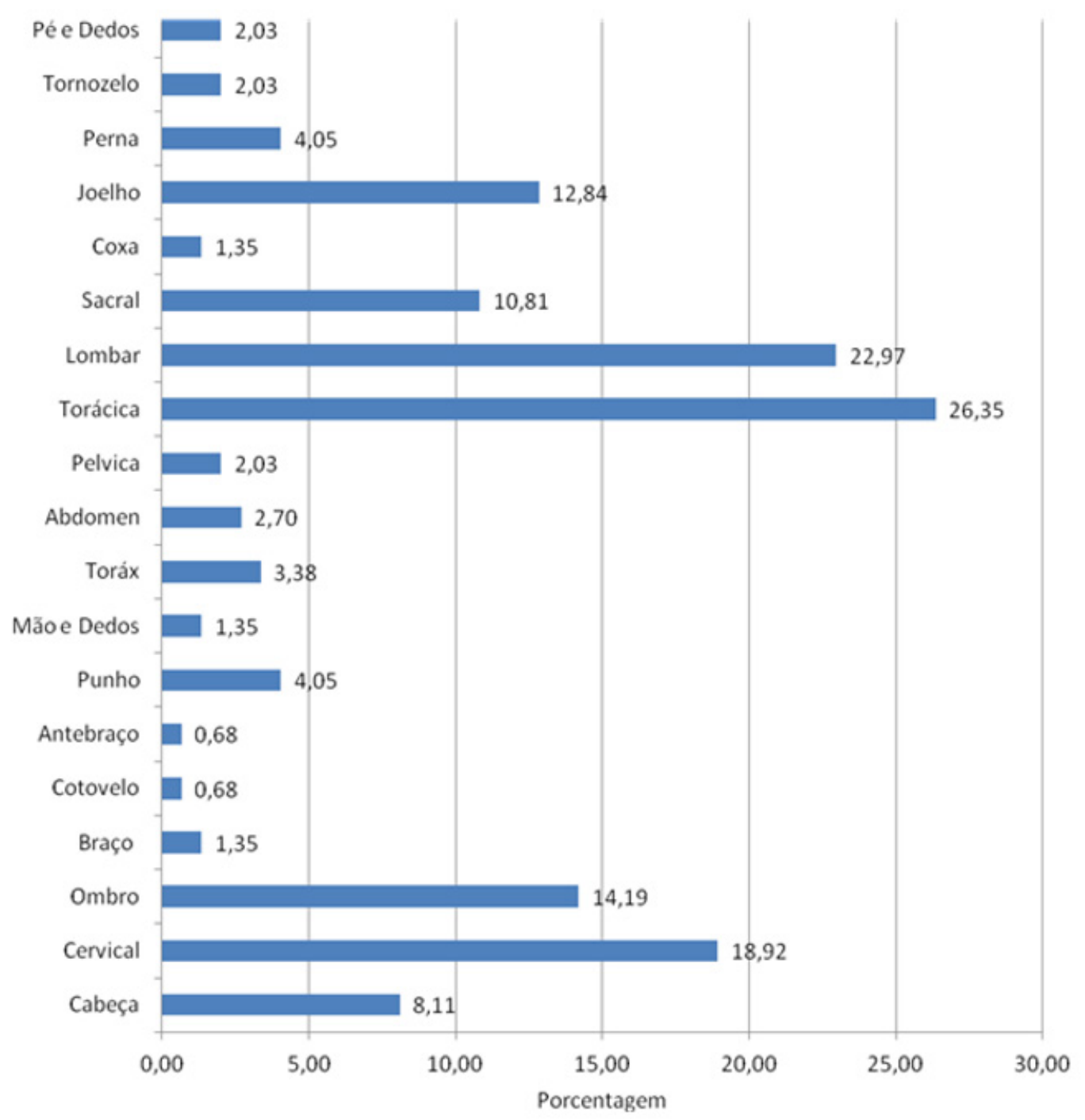

Gráfico 1. Avaliação de dores localizadas. 
média 1,17 pontos de dores. Os alunos do segundo ano de graduação apontaram as regiões da coluna torácica $(29,55 \%)$, coluna lombar $(27,27 \%)$ e coluna cervical $(22,73 \%)$, representando em média 1,50 pontos de dores. Em relação aos alunos do terceiro ano de graduação, as regiões mais apontadas foram coluna cervical (40\%), coluna lombar (40\%), joelho (20\%) e ombro (20\%), em média 1,50 pontos de dores. Entre os alunos do quarto ano de graduação, as regiões mais apontadas foram coluna torácica $(33,33 \%)$, ombro $(21,21 \%)$, coluna lombar $(21,21 \%)$ e sacral $(21,21 \%)$, apresentando em média 1,76 pontos de dores.

A região que mais prevaleceu entre todos os grupos foi a região torácica. Analisando tal região separadamente, observa-se um crescente conforme o tempo de experiência, porém os professores e os alunos do terceiro ano não foram de acordo com a hipótese por conta do número reduzido de voluntários, conforme mostrado no Gráfico 2.

Os alunos do último ano da graduação relataram mais regiões com dores do que os alunos dos anos anteriores, com 16 das 19 regiões do questionário, havendo a hipótese de que quanto mais tempo de atendimento clínico na graduação, maiores são as possibilidades de apresentarem dores no corpo.

\section{Nível de atividade física}

De acordo com a classificação do IPAQ, o nível de atividade física foi de $12 \%$ dos voluntários classificados como sedentários e 19,59\% apresentaram-se como irregularmente ativos B, $38,51 \%$ irregularmente ativos A, $24,32 \%$ como ativos e $4,73 \%$ classificados como muito ativos.

Quando comparados a incidência de dores e o nível de atividade física, observou-se que os indivíduos irregularmente ativos B foram os que mais relataram dor em duas ou mais regiões do corpo $(48,27 \%)$. Em relação ao grupo muito ativo, a incidência de dor em duas ou mais regiões foi menor $(28,57 \%)$. Na Tabela 2 podemos verificar os resultados obtidos em todos os grupos.

\section{Avaliação Biomecânica}

Os voluntários foram analisados por meio de filmagem e fotografia durante os procedimentos realizados. As regiões foram classificadas de acordo com o método RULA. Os valores dos ângulos apresentados foram adotados por maior parte do tempo de cada procedimento. Para a região cervical, o procedimento que apresentou um maior risco foi cimentação da coroa metalocerâmica. Para

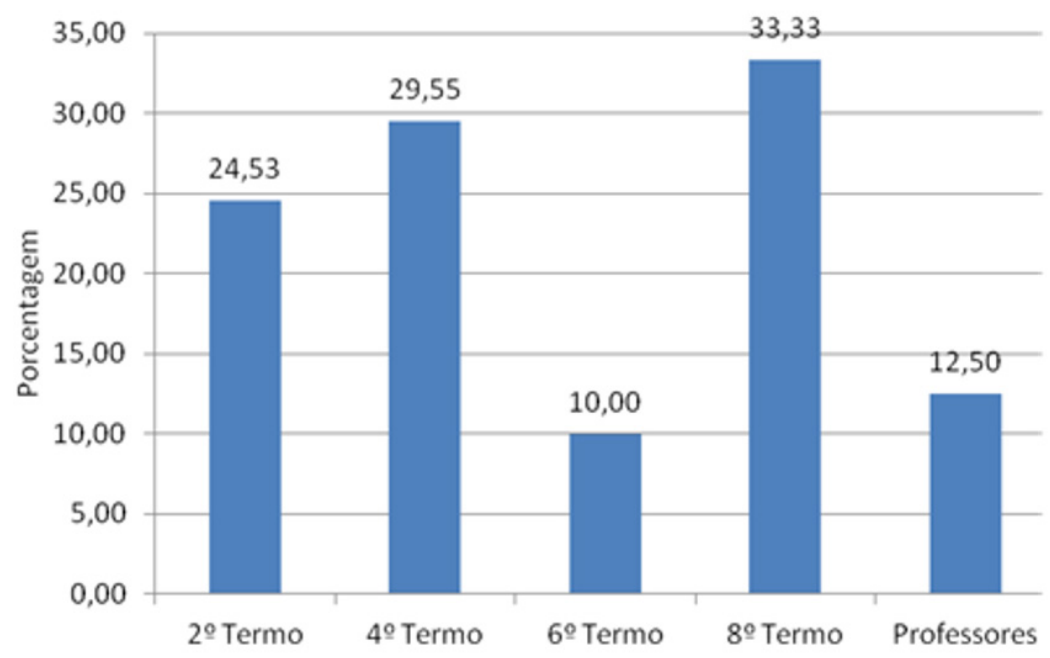

Gráfico 2. Dores localizadas na região torácica.

Tabela 2. Comparação entre o nível de atividade física e o nível de dores localizadas.

\begin{tabular}{cccccc}
\hline & Sedentário & $\begin{array}{c}\text { Irregularmente } \\
\text { Ativo B }\end{array}$ & $\begin{array}{c}\text { Irregularmente } \\
\text { Ativo A }\end{array}$ & \multirow{2}{*}{ Ativo } & \multirow{2}{*}{ Muito Ativo } \\
\hline Total de voluntários & 19 & 29 & 57 & 36 & 7 \\
Dor em 2 pontos ou mais & 8 & 14 & 26 & 11 & 2 \\
Porcentagem de dores & $42,11 \%$ & $48,28 \%$ & $45,61 \%$ & $30,56 \%$ & $28,57 \%$ \\
\hline
\end{tabular}


as regiões torácica e lombar, a instrumentação do canal radicular proporcionou um maior risco. Em relação à região do ombro, a obturação do canal radicular apresentou um maior risco de dores musculoesqueléticas. Região de cotovelo, procedimento de exodontia e para joelhos, periograma. Todos os dados obtidos podem ser observados na Tabela 3.

O score que mais prevaleceu entre todos os procedimentos foi o score 3. De acordo com a recomendação do RULA, esse score aponta que é necessário investigar e que intervenções podem ser necessárias.

\section{Discussão}

O ambiente e as condições de trabalho podem ser a causa de muitos problemas. Um dos fatores importantes são os distúrbios físicos relacionados ao trabalho que reduzem a produtividade. A Odontologia é uma profissão que muitas vezes provoca dores musculares. Hábitos de trabalho repetitivos de membros superiores, de longo prazo e associados com a necessidade de boa visibilidade são as causas de problemas. Essas dores geralmente são inofensivas e os sintomas geralmente são ignorados até que eles levem a um distúrbio crônico, provocando uma lesão permanente (Aghahi et al., 2018).

O presente estudo mostrou uma prevalência de dores localizadas, acentuada na região paravertebral (Ehsan et al., 2013), havendo uma inter-relação entre os anos de atividade laboral com o nível de dores apontados. Em um estudo realizado na Polônia com dentistas, $92 \%$ dos pesquisados tinham dores principalmente no pescoço e na região lombar. Houve também uma prevalência de dor no pescoço e nas costas em $88,4 \%$ dos dentistas no sudoeste da Nigéria (Saxena et al., 2013). Em Babol, no Iran, com 60 dentistas, a fim de investigar distúrbios musculoesqueléticos relacionados ao trabalho, constatou-se dores mais comuns no pescoço $(83,3 \%)$, cintura $(56,7 \%)$ e ombros (41\%) (Aram et al., 2015).

Tabela 3. Avaliação biomecânica.

\begin{tabular}{|c|c|c|c|}
\hline Procedimentos & Região & Graus & Scores \\
\hline \multirow{5}{*}{ Periograma } & Cervical & 56 & 3 \\
\hline & Torácica e lombar & 18 & 2 \\
\hline & Ombro & 7 & 1 \\
\hline & Cotovelo & 107 & 3 \\
\hline & Joelho & 98 & 2 \\
\hline \multirow{5}{*}{ Obturação } & Cervical & 38 & 3 \\
\hline & Torácica e lombar & 18 & 2 \\
\hline & Ombro & 37 & 2 \\
\hline & Cotovelo & 102 & 3 \\
\hline & Joelho & 84 & 2 \\
\hline \multirow{5}{*}{ Instrumentação do canal radicular } & Cervical & 71 & 3 \\
\hline & Torácica e lombar & 30 & 3 \\
\hline & Ombro & 6 & 1 \\
\hline & Cotovelo & 109 & 3 \\
\hline & Joelho & 77 & 2 \\
\hline \multirow{5}{*}{ Cimentação da coroa metalocerâmica } & Cervical & 100 & 4 \\
\hline & Torácica e lombar & 26 & 3 \\
\hline & Ombro & 9 & 1 \\
\hline & Cotovelo & 122 & 3 \\
\hline & Joelho & 88 & 2 \\
\hline \multirow{5}{*}{ Cirurgia } & Cervical & 79 & 4 \\
\hline & Torácica e lombar & 19 & 2 \\
\hline & Ombro & 18 & 1 \\
\hline & Cotovelo & 137 & 3 \\
\hline & Joelho & 85 & 2 \\
\hline
\end{tabular}


Entre os sintomas relatados, a dor cervical foi a mais alta $(88,17 \%)$, seguida da dor lombar $(86,02 \%)$ e no punho $(54,8 \%)$ (Batham et al., 2016). Dos distúrbios musculoesqueléticos relacionados ao trabalho na profissão odontológica, mostrou-se uma prevalência maior na região do pescoço $(83,8 \%)$ e ombro $(73,5 \%)$. O que deve ser levado em conta são a carga de trabalho e os fatores de estresse, com a implantação de uma abordagem de prevenção, intervenção e educação dos fatores de risco na Odontologia (Feng et al., 2014).

Em nosso estudo observou-se um baixo nível de atividade física entre os voluntários avaliados, indo ao encontro do estudo de Iwuala e colaboradores que fizeram uma pesquisa com 298 profissionais de diferentes áreas da saúde, entre eles dentistas, no Hospital Universitário de Lagos em Lagos na Nigéria. Os resultados mostraram que $79,02 \%$ dos estudados tinham um nível de atividade física baixo, 9,07\% nível moderado, $11,1 \%$ nível alto e $20,08 \%$ tinham o nível de atividade física adequado. Acredita-se que os fatores que contribuem para um nível baixo de atividade física entre os profissionais da área da saúde são as longas jornadas de trabalho e a natureza sedentária do trabalho (Iwuala et al., 2015).

As posturas de trabalho dos voluntários nesta pesquisa foram preocupantes por conta de alguns locais apresentarem prevalência de scores altos. Alguns estudos na literatura mostram avaliações posturais em dentistas, que apresentam um nível considerável de risco às lesões musculoesqueléticas. O método RULAé utilizado para avaliar as posturas dos dentistas. Foram avaliados e registrados os movimentos de diferentes partes do corpo durante 20 minutos. Os resultados que mostraram a maior porcentagem, $57 \%$ dos voluntários, se enquadraram nos scores 5 e 6, indicando investigação e mudanças necessárias em breve (Rafie et al., 2015). A postura de trabalho inadequada tem efeitos consideráveis sobre as lesões musculoesqueléticas. Investigações adicionais são necessárias.

\section{Conclusão}

A região paravertebral mostrou ser mais propícia às dores localizadas nos estudantes e professores. Indivíduos mais ativos fisicamente apresentaram um menor nível de dores em relação aos menos ativos.

\section{Referências Bibliográficas}

AGHAHI, R. H., DARABI, D., \& HASHEMIPOUR, M. A. (2018). Neck, back, and shoulder pains and ergonomic factors among dental students. Journal of Education and Health Promotion, 7, 40.

ARAM, T., KHODABAKHSH, J., ARASH, T., FATEMEH, A., \& ALIREZA, P. (2015). Musculoskeletal disorders among a group of Iranian general dental practitioners. Journal of Back and Musculoskeletal Rehabilitation, 28(4), 755-759.

BATHAM, C., \& YASOBANT, S. (2016). A risk assessment study on work related musculoskeletal disorders among dentists in Bhopal, India. Indian Journal of Dental Research, 27(1), 236-241.

DANTAS, F. F. O., \& LIMA, K. C. (2015). The relationship between physical load and musculoskeletal complaints among Brazilian dentists. Applied Ergonomics, 47, 93-98.

EHSAN, R., JAFARI, Z., KASHANI, F. O., \& RAHIMPOUR, F. (2013). A study on job postures and musculoskeletal illnesses in dentists. International Journal of Occupational Medicine and Environmental Health, 26(4), 615-620.

FENG, B., LIANG, Q., WANG, Y., ANDERSEN, L. L., \& SZETO, G. (2014). Prevalence of workrelated musculoskeletal symptoms of the neck and upper extremity among dentists in China. BMJ Open, 4, e006451.

GARCIA, P. S., \& CAMPOS, J. B. (2013). Risk of musculoskeletal disorders in upper limbs in dental students: concordance of different methods for estimation of body angle. Indian Journal of Dental Research, 24, 562-566.

IWUALA, S. O., OLAMOYEGUN, M. A., AKANBI, M.A., SABIR, A. A., \& AYANKOGBE, O. O. (2015). Self-reported physical activity among health care professionals in South-West Nigeria. Nigerian Journal of Clinical Practice, 18(6), 790-795.

MATSUDO, S., ARAÚJO, T., MARSUDO, V., ANDRADE, D., Andrade, E., Oliveira, L. C., \& Braggion, G. (2001). Questionário Internacional de Atividade Física (IPAQ): estudo de validade e reprodutibilidade no Brasil. Atividade Física \& Saúde, 6(2), 5-18. 
MCATAMNEY, L., \& CORLETT, E. N. (1993) RULA: A SURVEY METHOD FOR THE INVESTIGATION OF WORK-RELATED UPPER LIMB DISORDERS. APPLIED ERGONOMICS, 24(2), 91-99.

RAFIE, F., JAM, A. Z., SHAHRAVAN, A., RAOOF, M., \& ESKANDARIZADEH, A. (2015). Prevalence of upper extremity musculoskeletal disorders in dentists: symptoms and risk factors. Journal of Environmental and Public Health, 517346, 1-6.

SAXENA, P., GUPTA, S. K., \& JAIN, S. (2013). Work-related musculoskeletal pain among dentists in madhya pradesh, india: prevalence, associated risk factors, and preventive measures. Asia-Pacific Journal of Public Health, 26(3), 304-309.

SOUZA, I. M. A., VASCONSELOS, T. B., BASTOS, V.P. D., \& FARIAS, M. S. Q. (2012). Avaliação da dor e lesões ocasionadas pelo trabalho em cirurgiões-dentistas na cidade de Fortaleza/ CE. Revista Fisioterapia \& Saúde Funcional, 1(2), 35-41.

THAKAR, S., SHIVLINGESH, K. K., \& PRABHAKAR, I. (2015). High levels of physical inactivity amongst dental professionals: a questionnaire based cross sectional study. Journal of Clinical and Diagnostic Research : JCDR, 9(1), 43-46. 